\title{
A Novel Method for Identifying the Drowsiness while Driving
}

\author{
Hemantkumar B. Mali \\ Sinhgad College of Engineering, \\ Pune, Maharashtra
}

\author{
Shashikant D. Lokhande \\ Sinhgad College of Engineering, \\ Pune, Maharashtra
}

\begin{abstract}
This algorithm gives insight into the possible technique to recognize the state of the eye whether open or close. The algorithm explores the various features of the eyes when in closed and in open states. Using this feature, the decision is made whether the state of the eye is closed or open to detect drowsiness while driving.
\end{abstract}

\section{Keywords}

Close eye, Eye State, Eyelids, Iris, Open eye.

\section{INTRODUCTION}

This algorithm intends to recognize the state of the eye at any given time. The eye as a region of interest is extracted from the face. In real time the face can be detected using various established techniques [2]. The biggest problem regarding the increased traffic is the rising number of road accidents [3]. A system that will accurately monitor the open or closed state of the driver's eyes. It is believed that the symptoms of driver fatigue can be detected early enough to avoid a car accident by monitoring the eyes, Detection of fatigue involves a sequence of images of a face (capture in frames), and the observation of the eye blink rate and head position [4]. A vast amount of research is performed in the field of face and facial components detection, localization and tracking [5].

Care is taken to sample the image with specific resolution since the current version of the algorithm is partially static and needs a particular resolution eye image to proceed with the algorithm. The need for this will be explained in the further sections of this paper.

\section{REQUIRED IMAGE}

As mentioned earlier the input to the algorithm is the image of the eye. Firstly, the captured image/frame must be converted into the grayscale image if it is a color image. The RGB to GRAY conversion can be done by taking $30 \%$ of Red, $60 \%$ of Green and $10 \%$ of Blue. After extracting the face of the image using various image processing tools for the face extraction, the region of interest (ROI) must be extracted. Proper care is taken during this operation since the image of the eye (either left eye or right eye or both eyes in separate images) must have the resolution of $60 * 30$ pixels. This is required because the algorithm works by recognizing the exposed portion of the iris, which is analyzed by first cropping the new ROI with the help of the technique discussed ahead and then applying the algorithm.

\section{ENHANCING THE INPUT IMAGE}

The image of the eye that has its resolution $60 * 30$ is first processed using the histogram equalization technique. It is seen from the trials that this image enhancement technique increases the efficiency of the algorithm. Then the size of this image is stored in the variables ' $R$ ' and ' $C$ '. This is done to crop the image. Then the binary thresholding is done with an image. After lots of trials, it is found that during converting the image into the binary image, i.e. having only, two color levels 0 and 255, if the threading factor is taken as 70 then the $\mathrm{ROI}$ is enhanced desirably.

\section{METHODOLOGY}

\subsection{Cropping of Image}

After, converting the image into the binary format the next task is to crop the ROI to get a more desirable new ROI (henceforth referred nROI). This particular nROI has basically only the portion of the iris of the eye.

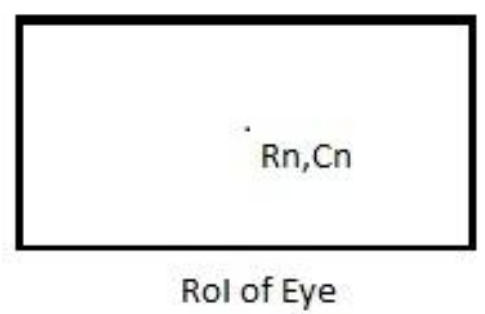

Figure 1. ROI of eye having resolution $60 * 30$

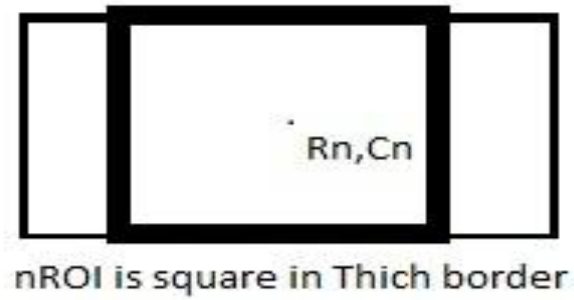

Figure 2. nROI of iris after Cropping

In crop image a specific formula is used,

$\mathrm{A}=\operatorname{crop}((\mathrm{A} 1,41$ [(Cn-15) (Rn-10) (30) (20)]) --- (1)

In Equation (1), ' $A$ ' signifies $n R O I$ and 'A1' signifies the ROI. It is as seen that the ' $\mathrm{Rn}$ ' and ' $\mathrm{Cn}$ ' are the centers of horizontal and vertical lengths respectively. This particular technique is used for cropping the image which has a new resolution of $30 * 20$ and mainly consist of the iris part of the image.

\subsection{Formation of Matrices 'ro' and 'co'}

In order to get the dimensions of the human eye's iris there is need to declare one blank row matrix to get the width of the iris, and need to declare the blank column matrix to get the height of the iris. Let these two matrices to be named 'ro (i,1)' for the blank row matrix and 'co $(1, \mathrm{j})$ ' for blank column matrix. It must be noted here that $\mathrm{i} * \mathrm{j}$ signifies the dimensions of the nROI. 
With the help of for loops traverse the image in the horizontal direction and calculate the number of black pixels present in each row and store this number in the respective element of the matrix 'ro'. Example, if the traversing 5th row of the image and the number of black pixels present are 6 , then in 'ro $(5,1)$ ' we need to store 6 .

Similarly, we need to traverse the image in a vertical manner and the number of black pixels present in each column must be stored in the respective column of the matrix 'Co'. Example, if we are traversing 7th column and the number of black pixels are 3 , then in 'Co $(1,7)$ ' we need to store 3 . It is described in the figure below.

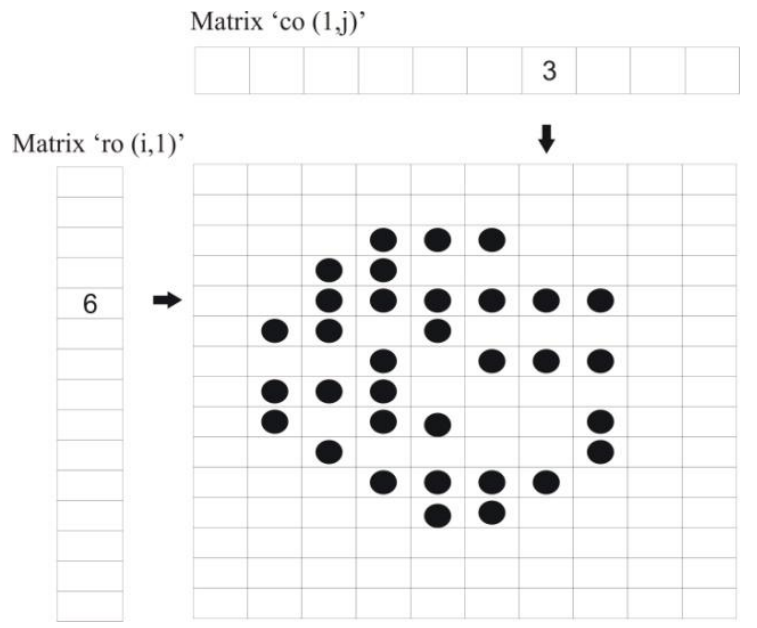

Figure 3. Illustration of image matrix with black pixels

As seen in the image the 5th row has 6 black pixels and the 7 th column has 3 black pixels. The matrix 'row' and ' $\mathrm{Co}$ ' storing these values in respective rows and columns.

\section{Length and Width of IRIS}

After all the values of matrices ro and co, analyze them to get the length or height and width of iris. As seen in figure 3, if all the values in ro are filled we get non-zero values from 3rd row up to 12 th row, rest all fields are zero since no black pixels are present in the respective rows. Similarly, in matrix co the nonzero fields ranges from 2 nd column up to 8 th column, rest all fields are zero since no black pixels are present in the respective columns.

Now if we assign a 4 variables, namely $r 1, r 2, c 1$ and c2 the values of first and last non-zero values of row and Co respectively, we get the following results $\mathrm{r} 1=3, \mathrm{r} 2=12, \mathrm{c} 1=$ 2 and $\mathrm{c} 2=8$

Now to get the height and width subtract $\mathrm{r} 1$ from $\mathrm{r} 2$ and store the value in ' $r$ ', and to get length subtract the $\mathrm{c} 1$ from $\mathrm{c} 2$ and store the value in ' $c$ '. Here, getting the height as $r$, and width as c.

\subsection{Concept}

The concept behind the algorithm is, after threshold the image into binary, getting a total black and white image. Here in the nROI irrespective of the closed or open eye the width of the iris will remain constant (note width of the iris signifies the black pixels spanning horizontally in nROI and not the actual width of the iris). This is due to the fact that that the eyelids in the image of the eye are generally black and are visible irrespective of closed or open eye or the extent of nROI cropped.
Also here the height of iris signifies the real height of iris since the human eye does not have any vertical feature that will give constant vertical black pixels when threshold, unlike that of the width of the iris. This very fact will help us determine the state of the eye.

Now iris are almost a circle. If compared the height and width of the iris and if they are almost equal or the height is greater than half of the width can safely say that the eye is open. This is because as mentioned earlier the height signifies the exposed iris in image, if the height is more than half that of width means the iris is seen in the image and hence the eye is open. But if the height is zero (ideal case, since due to thickness of eyelid height will never be zero, but very small) it means the iris is not seen in the image and thus the eye is closed.

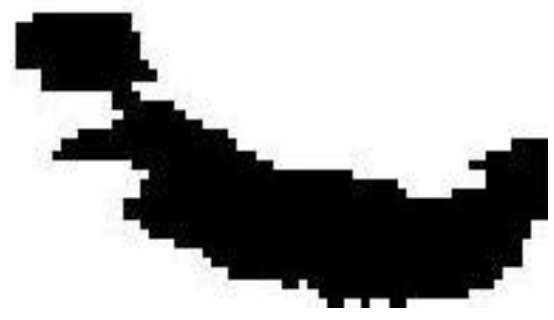

Figure 4. The nROI of the closed eye

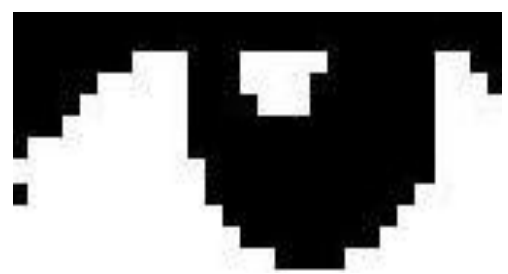

Figure 5. The nROI of the open eye

As seen in figure 4, the nROI consists only of the eyelids and the iris is not seen, hence concluded that the image is of closed eye. But in case if figure 5, the round iris is clearly seen, hence it is open eye.

\subsection{Ambiguity in Height of iris}

As in figure 5, height is more than half or nearly equal to the width of the iris hence it is clearly an open eye. But in figure 4, eyelids have a slant curve and due to this it undesirably increases the height of the iris. Due to this the algorithm may yield negative results. To solve this problem, develop the concept of continuity.

To elaborate the concept of continuity let us consider an two cases, in case one we have eyelids that are not curve and exactly straight, image having such an eyelid will follow the algorithm since the height will be very less than the width. But in case two where the eyelids in nROI are slant will increase the height of the image. It is to note that though both the cases give different height the thickness of the eyelid spanning from one end to the other is approximately constant. Hence, if we check if the height in co is approximately constant or the number of pixels throughout columns are nearly equal, thus getting the true state of the eye. The formulae derived that satisfy the previous two conditions (first checking the continuity and the second comparing iris height and width) are

$\mathrm{r}>\mathrm{c} / 2$; 
Compare the height and the width of the iris in equation (2) to check whether the eye is open or closed.

Median $(\operatorname{co})-\operatorname{co}(1,1)<3$;

In equation (3) take the median of the matrix co and thus get the approximate average of the heights of all columns and then subtract the height of the first column from this particular obtained height from the median function. If the difference is less than three then it means that the height is almost constant throughout and it qualifies the concept of continuity.

\section{RESULTS}

\subsection{Input Image.}

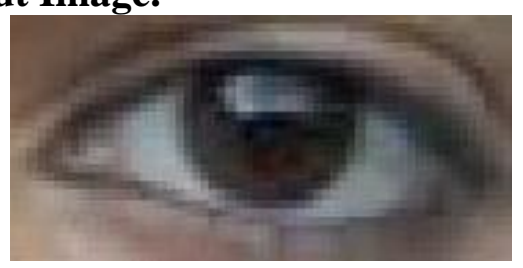

Figure 6. The ROI from image

The figure 6 , shows us the ROI of the inputted image itself measures $60 * 30$ pixels.

\subsection{Processed Image}

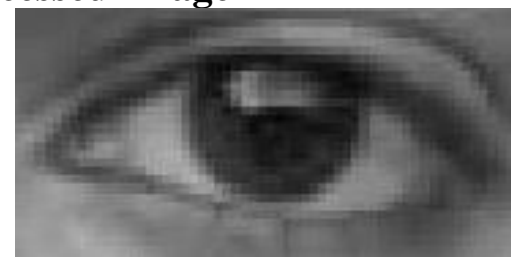

Figure 7. The image transformed into grayscale.

Figure 7, gives us the grayscale image of the color image that had given as input.

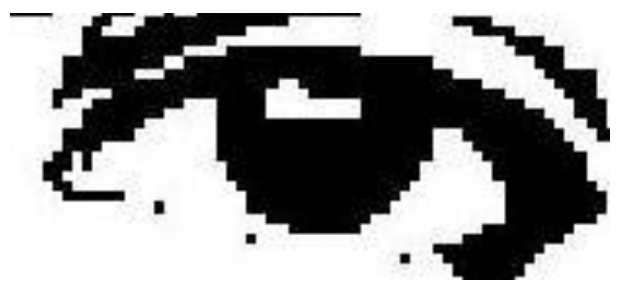

Figure 8. The image after thresholding and histogram equalizations.

The above figure 8 , gives us the image that has been processed with the histogram equalization technique and then subjected to the thresholding to covert the grayscale image into the binary image.

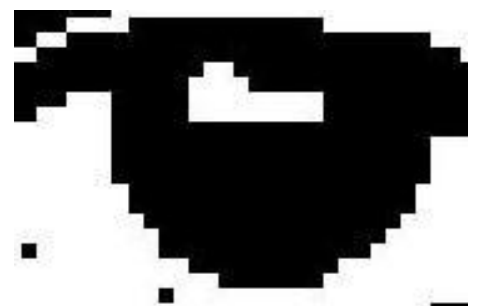

Figure 9. The nROI showing iris
Figure 9 is obtained after subjecting the figure 8 to equation (1) that is after cropping the image. The iris part of the human eye is significantly highlighted in the image.

The figure 9 is further required to determine the height and width of the iris and to check whether the eye is open or closed. In the above considered image the equation (3) is clearly violated hence it is not the close eye for sure.

Further, equation (2) yields,

$$
\text { - } \begin{aligned}
c & =30 \\
\text { - } \quad r & =24
\end{aligned}
$$

Hence, equation (2) is satisfied giving us clearly the result of OPEN EYE.

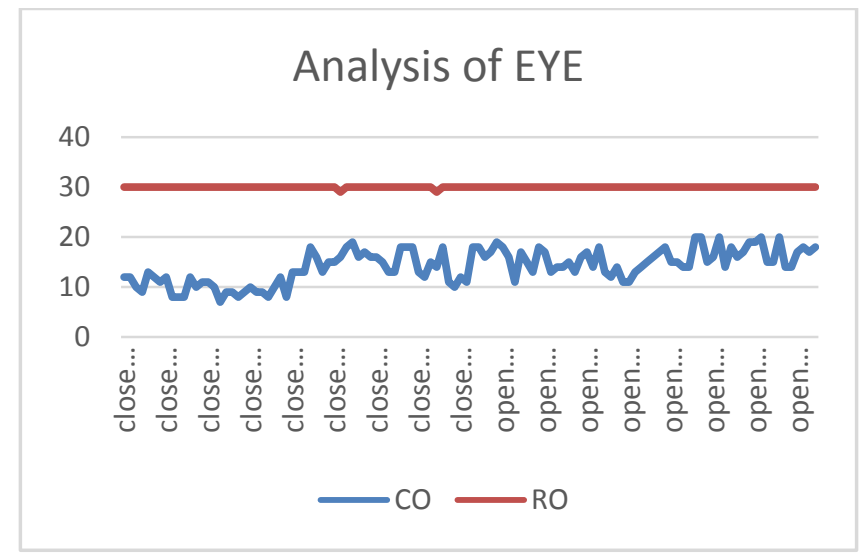

Figure 10. The Graph for Eye Analysis

The graph shows the values for 'co' and 'ro' for the different images of the eye. In the closed eyes, see that the 'co' is almost less than 15 , and which half of 'ro' that is 30 . The same can be shown for the open eye, which has the 'co' more than 15 . Hence this graph proves the equation (2).

Table1. Values of 'co' and 'ro' in various images

\begin{tabular}{|c|c|c|c|}
\hline & EYE & CO & RO \\
\hline 1 & close (1).jpg & 12 & 30 \\
\hline 2 & close (13).jpg & 13 & 30 \\
\hline 3 & close (17).jpg & 8 & 30 \\
\hline 4 & close (20).jpg & 10 & 30 \\
\hline 5 & close (24).jpg & 7 & 30 \\
\hline 6 & close (38).jpg & 18 & 30 \\
\hline 7 & close (39).jpg & 16 & 30 \\
\hline 8 & open (1).jpg & 18 & 30 \\
\hline 9 & open (10).jpg & 18 & 30 \\
\hline 10 & open (11).jpg & 16 & 30 \\
\hline 11 & open (12).jpg & 17 & 30 \\
\hline 12 & open (13).jpg & 19 & 30 \\
\hline 13 & open (14).jpg & 18 & 30 \\
\hline 14 & open (15).jpg & 16 & 30 \\
\hline 15 & open (16).jpg & 11 & 30 \\
\hline
\end{tabular}

The table shows values of 'co' and 'ro' for 15 images. The algorithm was implemented on 116 different images of close 
and open eyes. The summary of the implementation is in the table 2 .

Table 2.Summary of Images

\begin{tabular}{|c|c|c|c|c|}
\hline & $\begin{array}{c}\text { No. of } \\
\text { Images } \\
\text { of } \\
\text { Persons }\end{array}$ & $\begin{array}{c}\text { Correct } \\
\text { Detection }\end{array}$ & $\begin{array}{c}\text { False } \\
\text { Detection }\end{array}$ & $\%$ \\
\hline Open & 58 & 53 & 5 & 91.38 \\
\hline Closed & 58 & 50 & 8 & 86.21 \\
\hline
\end{tabular}

The efficiency obtained was $88.79 \%$.

\section{CONCLUSION}

After various conditions for checking the state of the eye. Need to check the conditions in a particular sequence to get proper results. First check the condition for continuity with equation (3), here itself, we will understand if the height is due to slant eyelids or iris. If it is due to slant eyelids we can say that eye is closed, but if it is due to iris then we check the condition in equation (2) and then we confirm that eye is closed or open. The efficiency obtained is $88.79 \%$ in the future, work can be carried out to enhance closed eye detection for more accuracy.

\section{REFERENCES}

[1] Rajat Garg, Veenet Agarwal and Vikrant Gupta, "A drowsy driver detection system and security systems," IEEE, 2009.

[2] Paul Viola and Michael Jones, "Rapid Object Detection using a Boosted Cascade of Simple Features," Computer vision and Pattern recognition, July 2001.

[3] Srinivasu and Praveen Kumar, "Driver Drowsiness Detection to Reduce the Major Road Accidents in Automotive Vehicles," International Research Journal of Engineering and Technology, Vol.2.Isssue 1, April 2015, pp. 345-349

[4] Neha Sharma and B. P. Lal, "Driver Drowsiness Detection Using Multi-feature Analysis," International Journal of Computer Science and Mobile Computing, Vol.4 Issue.7, July- 2015, pp. 426-431

[5] Ijaz Khan, Hadi Abdullah and Mohd Shamian Bin Zainal, "Efficient Eyes And Mouth Detection Algorithm Using Combination Of Viola Jones And Skin Color Pixel Detection", University Tun Hussein Onn Malaysia, June 2013. Volume 3, No. 4 